\title{
Gamma-ray Photons and Positrons from Type la Supernovae
}

\author{
Alina Hirschmann ${ }^{1,2}$, Eduardo Bravo ${ }^{1,2}$, and Jordi Isern ${ }^{2,3}$ \\ ${ }^{1}$ Departament de Fisica i Enginyeria Nuclear (UPC); Diagonal 647,08028 Barcelona (Spain) \\ ${ }^{2}$ Institut d'Estudis Espacials de Catalunya, Edifici NEXUS, c/Gran Capita 2, 08032 Barcelona \\ (Spain) \\ ${ }^{3}$ Institut de Ciencies de l'Espai (CSIC), Campus UAB, Fac. Ciencies, 08193 Bellaterra, \\ Barcelona (Spain)
}

E-mail: alina@ieec.uab.es, alina.hirschmann@upc.edu

\begin{abstract}
The explosion mechanisms of Type Ia Supernovae are still a matter of debate. Nevertheless, it is known that these systems produce considerable amounts of radioactive elements, which in turn produce gamma-ray photons and positrons. Therefore, the emission produced by these particles could provide valuable information on the explosion mechanisms and become an excellent diagnostic tool for the discernation between all the theoterical models proposed up to now.

In this paper we present gamma-ray simulations achieved by a 1D and 3D Monte Carlo code using a wide set of theoretical models. We study the behavior and transport of gamma-ray photons within the expanding ejecta by observing and analyzing the computed spectra and lightcurves of each model. Unfortunately, the theoretical predictions can not be compared with observations since there is no instrument sensitive enough to obtain high resolution data and be able to discern between models. New gamma-ray detectors are being proposed to reach this goal.

In addition to the gamma-ray analysis, positrons are also particles that play an important role in these events. A percentage of these escape from the explosion into the ISM and eventually annihilate giving rise to a $511 \mathrm{keV}$ emission line.
\end{abstract}

Supernovae: lights in the darkness (XXIII Trobades Científiques de la Mediterrània)

October 3-5 2007

Mao, Menorca, Spain 


\begin{tabular}{cccc}
\hline Models & Total Mass $\left(M_{\odot}\right)$ & ${ }^{56} \mathrm{Ni}$ Mass $\left(M_{\odot}\right)$ & Kin. Ene. $\left(10^{51}\right.$ ergs $)$ \\
\hline DDTa & 1.367 & 0.967 & 1.33 \\
DDTe & 1.367 & 0.511 & 1.04 \\
PDDa & 1.367 & 1.051 & 1.45 \\
PDDe & 1.367 & 0.521 & 1.12 \\
DD202c & 1.380 & 0.782 & 1.30 \\
W7 & 1.380 & 0.587 & 1.24 \\
\hline PRD6 & 1.40 & 0.70 & 1.15 \\
DDT3D & 1.42 & 0.64 & 0.82 \\
B3OU & 1.35 & 0.45 & 0.44 \\
\hline
\end{tabular}

Table 1: 1D and 3D model parameters. DDT: Delayed Detonation; PDD: Pulsating Delayed Detonation; DD202c: Delayed Det. provided by [10], W7: Deflagration provided by [17]; PRD6: Pulsating Reversed Detonation; DDT3D: Delayed Det.; B3OU: Deflagration

\section{Introduction}

Type Ia Supernovae (SN) are the thermonuclear explosion of a white dwarf, belonging to a binary system, that has approached the Chandrasekhar mass limit. In the outburst, radioactive elements are generated, which decay producing gamma-rays and positrons. The interaction of such particles with the ejecta is studied by analyzing the gamma-ray spectra and lightcurves of such events. Because there are no observations obtained up to now in this energy range, all the studies concerning this phenomenon are based on theoretical simulations. The simulations used for this work are based on a gamma-ray transport Monte Carlo code in 1D and 3D that has been built to reproduce the photon and positron transport within the expanding ejecta [7]. Table 1 shows the main input models for different proposed scenarios (deflagration, delayed detonation, pulsating delayed detonation, pulsating reversed detonation), provided by $[1,10,17]$ in $1 \mathrm{D}$ and $[4,3,5]$ in 3D. The code also provides an output gamma-ray line profiles, lightcurves and positron escape fractions.

Gamma-ray spectra give useful information on the internal structure of the system, abundances and location of radioactive isotopes and intermediate mass elements, age determination of supernova, and asymmetries in the explosion. Simulations in 1D provide a tool for determining the general properties of the explosion mechanism, however, since spherical symmetry is assumed, they cannot account for the rich phenomenology introduced by the existence of other freedom degrees. In 1D it is imposible to study and understand the effects produced by asymmetries in the ejecta. Such asymmetric effects along with the effects seen by the presence of the companion star are studied using the multidimensional code. In this paper we only present summarized results of simulations obtained with both codes but a detailed analysis of such results can be found in [9].

\section{Gamma-ray Emission}

The gamma-ray evolution is simulated through gamma ray spectra and lightcurves for a supernova explosion placed at a distance of $1 \mathrm{Mpc}$. Our results show that the $847 \mathrm{keV}$ line (from 


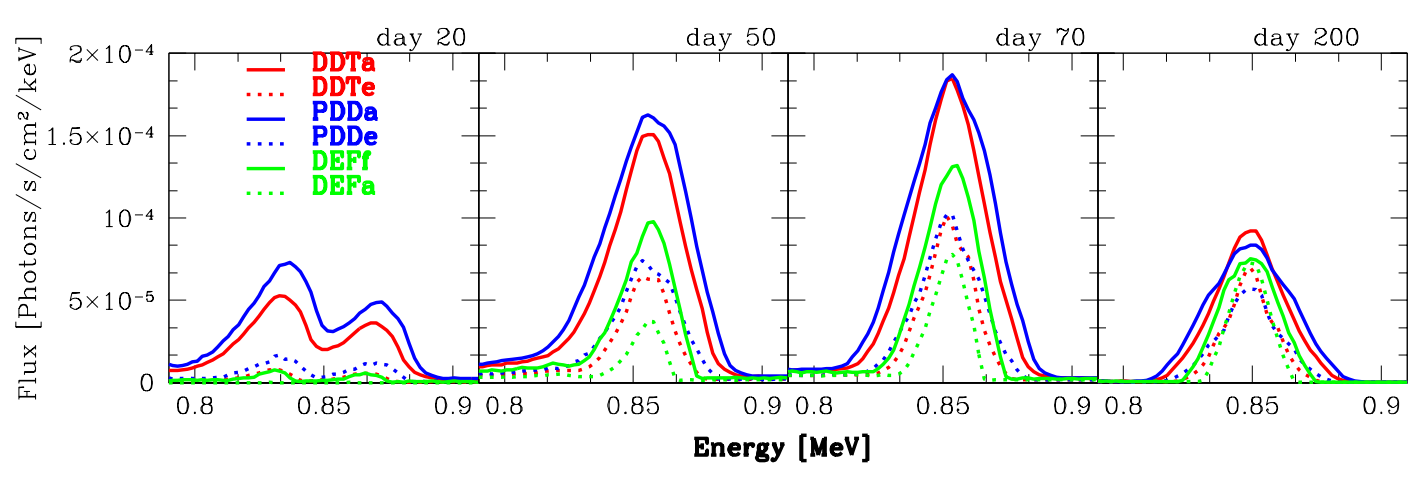

Figure 1: 1D spectrum of the $847 \mathrm{keV}$ emission line for the boundary cases of each model for different days after the explosion. This emission line is the strongest of the event.

${ }^{56} \mathrm{Co} \rightarrow{ }^{56} \mathrm{Fe}$ decay) is best suited for this end. Although this line is blended with the $812 \mathrm{keV}$ (for early epochs only) because high velocities of the ejecta create Doppler broadening, it is the strongest and most intense line seen in the spectra during the expansion phase.

\subsection{Spectra}

Many properties of the ejecta can be determined by measuring the width of the emission lines, such as the amount and location of radioactive elements. The evolution of the prominent $847 \mathrm{keV}$ line for the different subset of models is shown in Fig. 1. The intensity and broadness of the line is model dependent since it depends mainly on the expansion rate and the distribution of ${ }^{56} \mathrm{Ni}$. Most energetic models show a more intense and broader line while least energetic models show the opposite since larger kinetic energy means lower opacity at any given time. Approximately 200 days after the explosion, the transparency of the medium allows the line profiles to reveal the distribution, in space velocity, of the parent radioactive elements in the ejecta. The truncated top of gamma-ray lines (i.e.pulsating delayed detonation models) reflects the lack of the ${ }^{56} \mathrm{Ni}$ isotope in the central regions of the star.

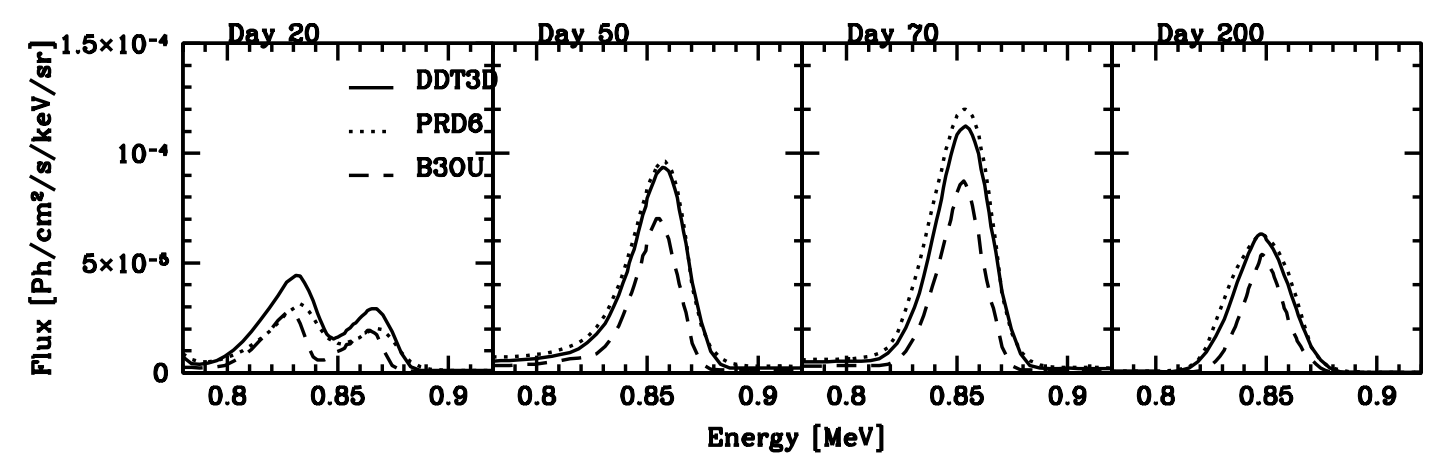

Figure 2: 3D spectrum of $847 \mathrm{keV}$ emission line for models DDT3D, PRD6 and B3OU for different days after the explosion, averaged over all directions 

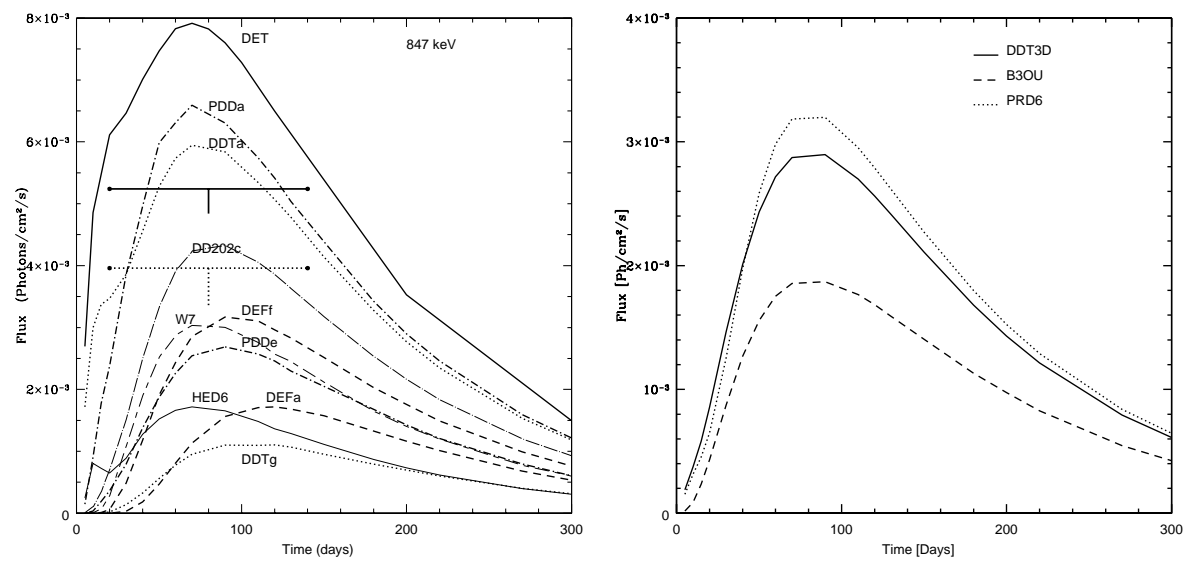

Figure 3: Left Panel:Lightcurves of all 1D models (extreme cases of submodels) for the $847 \mathrm{keV}$ line. The two constant lines plotted in the graph display upper limit integrated fluxes obtained by [6] for SN 1998bu with Comptel. These lines represent upper limits obtained using two different methods: spectral method (solid line) and imaging method (dotted line). Lines for model subsets: DEF (dashed), DDT (dotted), PDD (dot-dahsed), DD202c (long dot-dashed), W7 (longdashed), DET (thick solid), and HED6 (thin-solid). Right Panel: Lightcurve of emission line $847 \mathrm{keV}$ for 3D models: DDT3D, PRD6 and B3OU. The lines denote the integrated flux over the entire sphere. NOTE: vertical scales are different for both graphs.

In 3D, the spectra of the integrated flux over the entire sphere for all models are plotted in Fig. 2. None of the lines present truncated tops, which indicates that there is a non-negligible amount of ${ }^{56} \mathrm{Ni}$ in the central regions of the ejecta.

\subsection{Lightcurves}

Figure 3 includes the only observational upper limits ever obtained from a SNIa in gammarays, SN 1998bu (at a distance of 11.3 Mpc), using COMPTEL [6]. The two plotted upper limits are derived from two different methods imployed to reduce the background noise as much as possible. Evidently, the graph shows that it is very difficult to discern between models because families of models overlap and the observational upper limits do not provide sufficient information to constrain the actual flux from the supernova. The only model that can be discarded is the pure detonation DET since it does not agree with the measurements (its flux is beyond the upper limits).

In 3D, the lightcurves follow the same trend as in 1D and have their intensity curves comprehended within the same family of models in 1D (i.e. DDT3D's intensity is comprehended within the DDTa-DDTg gap) (see right panel Fig. 3). The peak luminosity curve for some 3D models tends to occur slightly earlier in time since the distribution of ${ }^{56} \mathrm{Ni}$ in $1 \mathrm{D}$ models is assumed to be stratified while in 3D it is well mixed within the ejecta. Hence, the luminosity evolution could be a good way of discriminating between well-mixed ejecta models and spherically symmetric ones if the SNIa explosion occurs close enough to measure the line flux for a long period of time.

\section{Positron Escape Fractions}

Positrons are generated from the beta decay of radioactive elements. The majority of these 
particles come from the decay chain ${ }^{56} \mathrm{Co} \longrightarrow{ }^{56} \mathrm{Fe}$. Once a positron is generated it will travel within the expanding ejecta, with the possibility of reaching the surface and escaping to the ISM or being absorbed. If the latter occurs, the particle will suffer interactions causing it to lose energy as it moves through the ejecta, eventually becoming thermalized in the medium and annihilating, giving rise to the local $511 \mathrm{keV}$ emission line of the supernova. The energy loss mechanisms for positrons are described in [2]. Ionization and excitation of the medium is the dominant process. Our code does not provide a thermal state of the expanding medium. However, the ionization state is well constrained by the optical spectra. Therefore, two ionization cases are considered: a triple ionized medium $\left(\chi_{e}=3.00\right)$, and a one percent ionization medium $\left(\chi_{e}=0.01\right)$. In all these calculations, a radial magnetic field is considered in accordance to [16] eventhough there are several observational studies that favour an entangled field configuration [20]. Observations presented by [21] and [13] in the optical and infrared are not able to completely assure that total deposition of positron energy occurs in the late-time ligthcurves of these events.

\begin{tabular}{c|cccccc|ccc}
\hline \hline \multicolumn{10}{c}{$N_{e^{+}}\left[10^{53} e^{+} /\right.$event $]$} \\
\hline Ionization & \multicolumn{10}{c}{ 1D models } \\
States & PDDa & PDDe & DDTa & DDTe & W7 & DD202c & PRD6 & B3OU & DDT3D \\
$\chi_{e}=0.01$ & 4.809 & 1.529 & 3.602 & 0.905 & 1.658 & 2.924 & 2.379 & 1.526 & 2.613 \\
$\chi_{e}=3.00$ & 3.583 & 1.004 & 2.542 & 0.629 & 1.038 & 2.000 & 1.572 & 0.961 & 1.853 \\
\hline \hline
\end{tabular}

Table 2: Positron production rate for several $1 \mathrm{D}$ models and 3D models. Values in the first row correspond to $1 \%$ ionization medium while values in the second row are for atriple ionization medium.

The escape fractions for the input models are displayed in Table 2. The average escape fraction of all $1 \mathrm{D}$ and $3 \mathrm{D}$ models results in $7.2 \pm 1.0 \%$ taking into account the expected occurence weights assigned to each model in the case of being a sub (16\%), super (20\%) and normal SNIa (64\%) [14]. This average escape fraction implies an accumulated value of $N_{e^{+}}=2.1 \times 10^{53} e^{+} /$event. This value can be relevant to account the positron annihilation emission line seen in the galactic center and disk by INTEGRAL ([23]).

\section{Conclusions and Remarks}

Radioactive elements play a crucial role in SNeIa. They provide valuable information that can determine the explosion mechanism. The only observations up to now in gamma of a SNIa (SN1998bu with COMPTEL) have provided two upper limits that are not able to discern between theoretical models. New gamma ray detectors [22], with sensitivities at least two orders of magnitud higher than INTEGRAL, need to be built in order to reach detection distances up to $\sim 50 \mathrm{Mpc}$. Gamma-ray observation up to this distance will provide a larger data set of SNIa which is needed in order to determine the general explosion mechanism of such events. As for the positron production and annihilation rates, the theoretical-observational comparisons favour the idea that SNIa could be one of the possible sources of the galactic $511 \mathrm{keV}$ emission observed with INTEGRAL. 


\section{References}

[1] Badenes C., Bravo E., Borkowski K. \& Dominguez I, 2003, ApJ, 593,358

[2] Chan, K. and Lingenfelter, R., 1993, ApJ, 405, 614

[3] Garcia-Senz, D. and Bravo, E., 2003, 158, http://adsabs.harvard.edu/abs/2003fthp.conf..158G

[4] Garcia-Senz, D. and Bravo, E., 2005, A \& A, 430, 585-602

[5] Bravo, E. and Garcia-Senz, D., 2006, ApJ, 642, L157-L160

[6] Georgii et al., 2002, A \& A, 394, 517G

[7] Gomez-Gomar,J. et al., 1998, MNRAS, 295,1-9

[8] Higdon, et al., 2007,astro-ph/0711.3008

[9] Hirschmann, A. et al. 2008, in preparation

[10] Hoflich P., Khokhlov A., \& Muller E., 1992, A\&A, 259,549

[11] Hoflich, P., et al., 2005, AAS, 37,468

[12] Knodlseder et al, 2005, A\&A, 441, 513K

[13] Lair et al, 2006, NewAstroRev, 50, 570-573

[14] Li et al., 2001, ApJ, 546, 734

[15] Mannucci et al., 2004, astro-ph/0411450

[16] Milne P., The L-S. \& Leising M., 1999, ApJS, 124,503

[17] Nomoto K., Thielemann F.-K. \& Yokoi K., 1984, ApJ, 286,644

[18] Prantzos N., 2006,A \& A, 449, 869

[19] Robin et al., 2004, astro-ph/0401052

[20] Sollerman et al., 2004, A\&A, 428,555

[21] Stritzinger, M. and Sollerman, J., 2007, A \& A, 470, L1-L4

[22] von Ballmos P., et al.,2005, in The 5th INTEGRAL Workshop on the INTEGRAL Universe, Ed. V. Shonfelder, G. Lichty and G. Winkler (ESA SP 552), p.747

[23] Weidenspointner, G. et al., 2007, astro-ph/0702621 